ARTICLE

DOI: $10.1038 / \mathrm{s} 41467-017-02245-1$

\title{
Loss of PBRM1 rescues VHL dependent replication stress to promote renal carcinogenesis
}

\author{
Judit Espana-Agusti ${ }^{1,6}$, Anne Warren ${ }^{2}$, Su Kit Chew ${ }^{3,4}$, David J. Adams ${ }^{5}$ \& Athena Matakidou (D) ${ }^{1,7}$
}

Inactivation of the VHL (Von Hippel Lindau) tumour suppressor has long been recognised as necessary for the pathogenesis of clear cell renal cancer (ccRCC); however, the molecular mechanisms underlying transformation and the requirement for additional genetic hits remain unclear. Here, we show that loss of VHL alone results in DNA replication stress and damage accumulation, effects that constrain cellular growth and transformation. By contrast, concomitant loss of the chromatin remodelling factor PBRM1 (mutated in $40 \%$ of ccRCC) rescues VHL-induced replication stress, maintaining cellular fitness and allowing proliferation. In line with these data we demonstrate that combined deletion of $\mathrm{Vhl}$ and $\mathrm{Pbrm} 1$ in the mouse kidney is sufficient for the development of fully-penetrant, multifocal carcinomas, closely mimicking human ccRCC. Our results illustrate how VHL and PBRM1 co-operate to drive renal transformation and uncover replication stress as an underlying vulnerability of all VHL mutated renal cancers that could be therapeutically exploited.

\footnotetext{
${ }^{1}$ Department of Oncology, University of Cambridge, CRUK Cambridge institute, Cambridge CB2 ORE, UK. ${ }^{2}$ Department of Pathology, Addenbrooke's Hospital, Cambridge CB2 OQQ, UK ${ }^{3}$ Cancer Genome Project, Wellcome Trust Sanger Institute, Hinxton CB10 1SA, UK ${ }^{4}$ Translational Cancer Therapeutics Laboratory UCL Cancer Institute, London WC1E 6DD, UK ${ }^{5}$ Experimental Cancer Genetics, Wellcome Trust Sanger Institute, Hinxton CB10 1SA, UK ${ }^{6}$ Present address: Medlmmune, AstraZeneca, Cambridge CB21 6GH, UK ${ }^{7}$ Present address: Centre for Genomics Research, IMED Biotech Unit, AstraZeneca, Melbourn SG8 6HB, UK Correspondence and requests for materials should be addressed to A.M. (email: Athena.Matakidou@cruk.cam.ac.uk)
} 
$\mathrm{R}$ enal cancer is the twelfth commonest cancer worldwide, accounting for 338,000 new cases and 143,000 cancerrelated deaths a year ${ }^{1}$. Around $50 \%$ of all patients develop metastatic disease and in this setting, renal cancer remains incurable with a median survival of around 28 months ${ }^{2}$. The development of effective treatment regimens has been hampered by a limited understanding of the basic molecular pathways underpinning renal cell carcinoma (RCC) carcinogenesis and the lack of genetically relevant animal models.

Clear cell RCC (ccRCC), the commonest histological subtype, is characterised by the inactivation of $V H L$ tumour suppressor gene $^{3}$. VHL is responsible for the ccRCC-predisposing syndrome, von Hippel-Lindau ${ }^{4}$ and is inactivated by either mutation or methylation in over $80 \%$ of sporadic cases ${ }^{5-7}$. The second allele is lost as a part of large $3 \mathrm{p}$ deletions, observed in $~ 90 \%$ of all cases ${ }^{8-}$ 10. Chromosome $3 p$ loss and VHL mutation or methylation, are early, truncal events during RCC development ${ }^{11}$ and therefore considered crucial for renal transformation.

VHL is implicated in a number of cellular functions, disruption of any or all of which could contribute to carcinogenesis. These include mediation of adaptive responses to hypoxia (through activation of hypoxia inducible factors HIF1a and HIF2a), extracellular matrix assembly, ciliogenesis, microtubule stabilisation, senescence, and DNA repair ${ }^{3}, 12$. Current data, however, suggest that $V H L$ loss alone, though necessary, is not sufficient for tumour initiation in the kidney. First, kidneys of patients with familial VHL disease typically contain many thousands of sites of biallelic VHL loss, with ccRCC arising relatively infrequently ${ }^{13}$. Second, large-scale sequencing efforts have shown that biallelic intragenic VHL mutations are not observed in sporadic ccRCC but instead one copy of the gene is always lost as a part of chromosome $3 p$ deletion, simultaneously affecting a number of other genes ${ }^{11,14}$. Third, deletion of $V h l$ or activation of its main downstream effectors (Hifla/Hif2a) within the mouse kidney does not lead to tumour development ${ }^{15-19}$. These observations suggest the requirement of additional genetic events to drive transformation.

Recently, next-generation sequencing studies have identified the SWI/SNF chromatin remodeller PBRM1 as the second most frequently mutated ccRCC gene ${ }^{10}, 14$. Concomitant loss of $V H L$ and PBRM1 is observed in up to $40 \%$ of all cases ${ }^{14}$ and phylogenetic analyses have suggested that $P B R M 1$ may act as a renal cancer driver as mutations are frequently acquired early ${ }^{11}$. Here we set out to ascertain the molecular interactions between VHL and PBRM1, and their potential to drive renal carcinogenesis in vivo. Our data show that loss of VHL alone induces replication stress and DNA damage accumulation, responses that curtail cellular proliferation and transformation. Significantly, the concomitant loss of PBRM1 rescues VHL-dependent replication stress, conferring a survival and proliferative advantage and thereby permitting renal carcinogenesis. Our study provides essential insights into the molecular mechanisms underpinning renal transformation and highlights replication stress as an underlying vulnerability of ccRCC.

\section{Results}

PBRM1 loss rescues VHL-induced replication stress. To identify interactions arising from the concurrent loss of VHL and PBRM1 within the context of normal cellular physiology, we generated primary mouse embryonic fibroblasts (MEFs) from either wild-type (WT), Vht $f^{l / f l}, \mathrm{Pbrm}^{\mathrm{fl} / f l}$ or double-mutant $\left(V h f^{\mathrm{fl}}\right.$ $\left.f_{;} P b r m 1^{f l / f l}\right)$ conditional mice. Recombined MEFs are hereafter referred to as $\mathrm{Vhl}^{-/-}, \mathrm{Pbrm}^{-/-}$and $\mathrm{Vhl}^{-/-} ; \mathrm{Pbrm}^{-/-}$(Supplementary Fig. 1a,b). To replicate physiological tissue conditions ${ }^{20}$ and avoid the well-documented sensitivity of MEFs to $21 \%$ oxygen $^{21,22}$, all experiments were performed at $5 \% \mathrm{O}_{2}$. As both VHL and PBRM1 have been previously implicated in DNA repair and the maintenance of genome stability ${ }^{12,23-25}$, pathways essential for tumour suppression, we decided to investigate their combined effect on these processes.

Analysis of DNA damage markers demonstrated significant accumulation of $\gamma \mathrm{H} 2 \mathrm{AX}$ and 53BP1 foci in late (day 20 of culture) vs. early (day 8) $\mathrm{Vhl}^{-/-}$cells (Fig. 1a and Supplementary Fig. 1c), indicating accrual of DNA damage upon replication. Surprisingly, this phenotype was restricted to $\mathrm{Vhl}^{-/-}$cells with $\mathrm{Vhl}^{-1-} ; \mathrm{Pbrm} 1$ $-/-$ MEFs displaying no significant damage accumulation. This disparity was not attributable to differences in the proliferation or senescence rates between the two groups (Supplementary Fig. 1d, e), suggesting that the rescue of this $V h l$-induced phenotype was mediated by loss of Pbrm1.

The accumulation of DNA damage markers in Vhl-deleted cells is indicative of the presence of replication stress. Replication stress broadly defines impediments in DNA replication, including stalling and collapse of replication forks and activation of the DNA damage response primarily mediated by the kinase ATR (ATM- and Rad3-related ${ }^{26}$. Indeed, late passage $\mathrm{Vhl}^{-1-}$ MEFs displayed significant activation of both ATR and its effector kinase CHK1 (Fig. 1a and Supplementary Fig. 1c). To investigate whether VHL loss induces replication stress, MEFs were assayed for hypersensitivity to mitomycin $\mathrm{C}$ (MMC) and aphidicolin $(\mathrm{APH})$, agents which result in replication fork stalling. $\mathrm{Vhl}^{-/-}$ cells displayed significantly higher levels of DNA damage markers upon treatment with these agents compared to non-recombined controls (Fig. 1b and Supplementary Fig. 2). This phenotype was rescued by concomitant treatment with cyclin-dependent kinase inhibitors to halt S-phase progression (Fig. 1c), suggesting the presence of a replication-dependent defect.

To ascertain the relevance of these observations within differentiated renal tubular epithelia (the presumed cell of origin of human ccRCC) $)^{27-29}$, we sought to generate genetically engineered mice with renal tubular specific deletion of $V h l$, Pbrm 1 or both $V h l$ and Pbrm1. We have previously described the development of Pax $8 \mathrm{CreER} \mathrm{R}^{T 2}$ transgenic mice ${ }^{16}$ that allow highly specific and inducible targeting of all renal tubular compartments (proximal, distal tubules and collecting ducts). Pax8CreER ${ }^{T 2}$ transgenic mice were crossed with Vhtlffl, Pbrmi ${ }^{f l / f l}$ and $V h f^{f l / f l}$; Pbrm $1^{f l f l}$ conditional animals to generate compound mutants.

Tamoxifen administration induced deletion of target genes to generate experimental $\left(\mathrm{Vhl}^{-/-}, \mathrm{Pbrm}^{-/-}\right.$and $\left.\mathrm{Vhl}^{-/-} ; \mathrm{Pbrm}^{-1-}\right)$

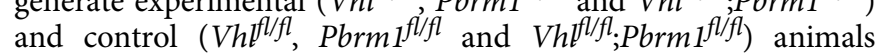
(Supplementary Fig. 3a,b). Mice were treated with MMC and their kidneys were assayed for markers of DNA damage. Post MMC treatment, kidneys from $\mathrm{Vhl}^{-/-}$animals demonstrated a threefold increase in the number of damaged tubular epithelia and induction of cell cycle arrest (Fig. 1d and Supplementary Fig 3c). Within individual animals, the extent of observed DNA damage closely correlated with the degree of VHL deletion, as assayed by CA9 positivity (Supplementary Fig. 3d). Significantly, $\mathrm{Vhl}^{-1-} ; \mathrm{Pbrm}^{-/-}$mice did not display hypersensitivity to MMC nor $\gamma \mathrm{H} 2 \mathrm{AX} / \mathrm{CA} 9$ correlation, despite displaying an equivalent extent of VHL loss to $\mathrm{Vhl}^{-/-}$cohorts (Supplementary Fig. 3d). Altogether, these data show that, both in vitro and in vivo, loss of VHL induces replication stress that is rescued upon concomitant loss of PBRM1.

Vhl loss induces replication fork instability and collapse. To directly explore the effect of $V h l$ inactivation on DNA replication, we evaluated replication fork dynamics using DNA fibre assays. Under unperturbed conditions, we observed no differences in replication fork progression and sister fork symmetry (Fig. $2 \mathrm{a}$ and 
$\mathbf{a}$
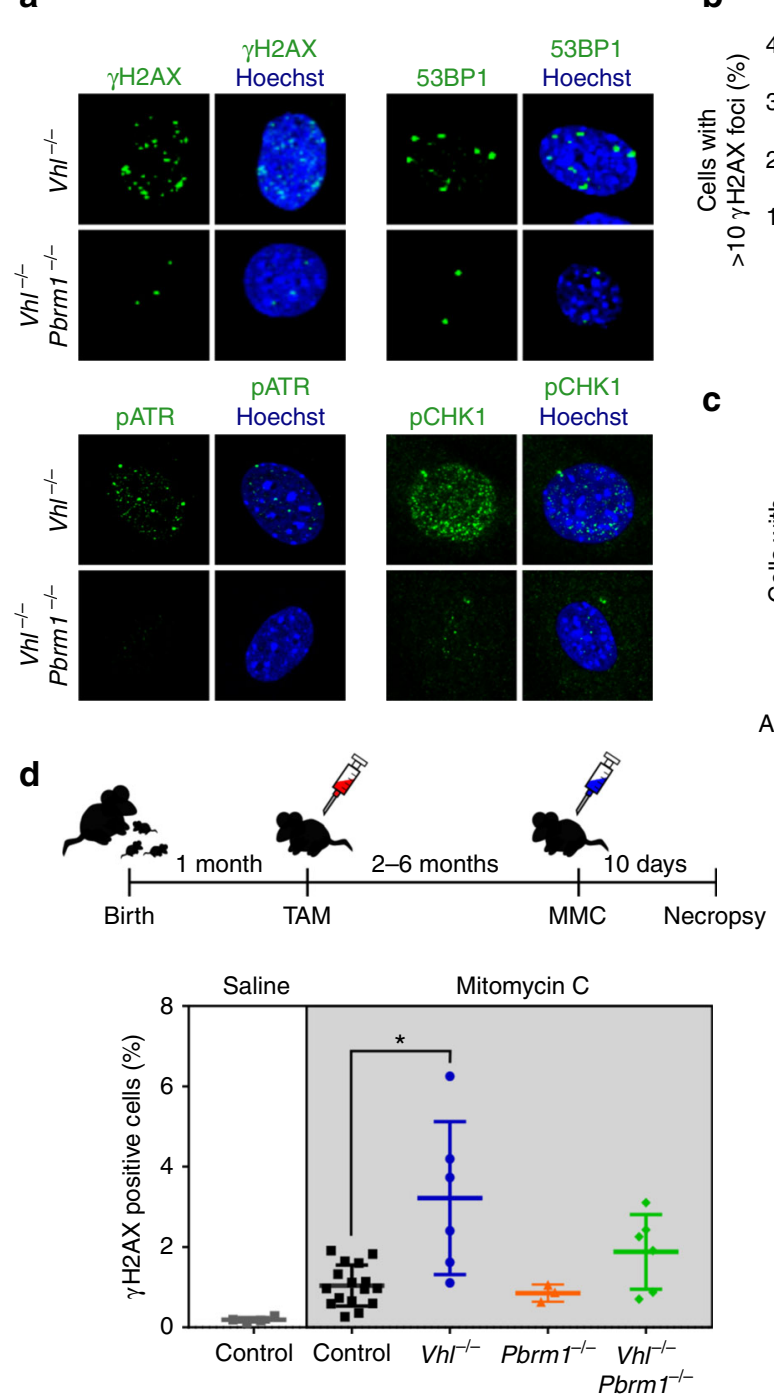

c
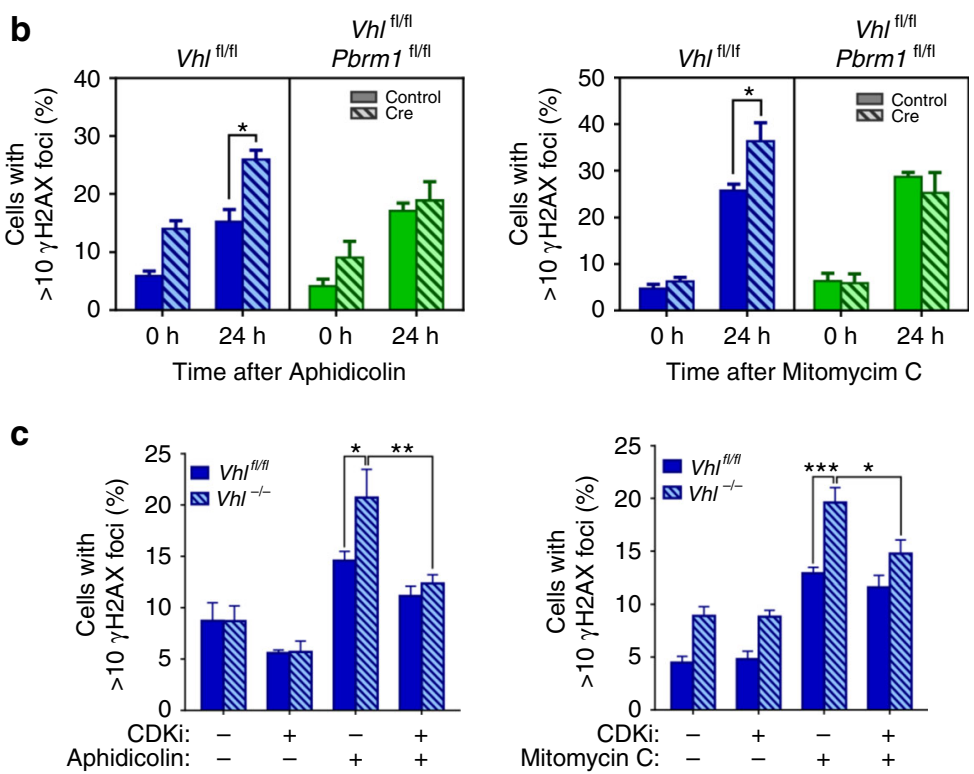

$\gamma \mathrm{H} 2 \mathrm{AX}$

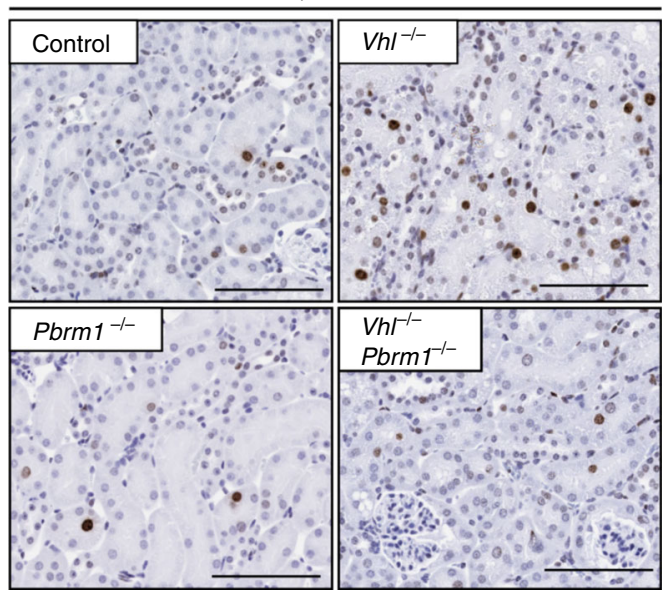

Fig. 1 Loss of VHL induces replication stress rescued by concomitant loss of PBRM1. a Representative photos of immunofluorescence staining in day 20 recombined MEFs. b, c Quantification of $\gamma \mathrm{H} 2 \mathrm{AX}$ foci formation following treatment of MEFs as indicated (b, $n$ (independent experiments) $=3$; $\mathbf{c}$, left, $n$ (independent experiments) $=6$; right, $n$ (independent experiments) $=4$ ). $\mathbf{d}$ Schematic of experimental design of in vivo Mitomycin $C(M M C)$ experiment; renal $\gamma \mathrm{H} 2 \mathrm{AX}$ quantification following MMC and representative immunohistochemical photos ( $n$ (mice); Saline: $\operatorname{control}=6 ; \mathrm{MMC}$ : $\mathrm{control}=16 ; \mathrm{Vh}{ }^{-/-}=6$; $\mathrm{Pbrm}^{-/-}=3 ; \mathrm{Vhl}^{-/-} ; \mathrm{Pbrm}^{-/-}=7$ ). TAM: tamoxifen. Scale bars, $100 \mu \mathrm{m}$. b, c Graphs depict means \pm s.e.m. (error bars); two-way ANOVA, Sidak's correction. d, Graph depicts means \pm s.d. (error bars); Kruskall-Wallis, Dunn's correction. ${ }^{\star \star \star} p<0.001,{ }^{\star \star} p<0.01,{ }^{\star} p<0.05$

Supplementary Fig. 4a). However, upon treatment with aphidicolin, $\mathrm{Vhl}^{-/-}$MEFs showed impaired fork progression and evidence of sister fork asymmetry consistent with fork stalling (Fig. 2b and Supplementary Fig. 4b), implicating VHL in the maintenance of DNA synthesis under conditions of replicative stress.

Following on from these observations we sought to determine how the loss of VHL may impact on DNA replication. The best studied function of VHL is its role in cellular oxygen sensing ${ }^{3}$. Loss of VHL results in activation of cellular responses to hypoxia despite normal oxygen conditions. Hypoxia has been implicated in replication stress and the potentiation of genomic instability through transcriptional and/or translational down regulation of DNA repair pathways ${ }^{30-32}$. We, therefore, hypothesised that the pseudohypoxia induced by VHL loss might have a similar effect. Indeed, VHL depleted MEFs showed a significant reduction in the levels of RAD51 and replication protein A (RPA32), two essential regulators of the replication stress response ${ }^{33,34}$ (Fig. 2c and Supplementary Fig. 4c). We observed no change in RAD51 and RPA32 transcript levels suggesting that transcriptional repression does not significantly account for the observed depletion of RAD51 and RPA upon loss of VHL (Supplementary Fig. 4d). As both RAD51 and RPA have essential roles in preventing the degradation of stalled forks and ensuring protection of nascent DNA $^{35,36}$ we used DNA fibre assays to investigate fork stability in the context of $V h l$ deletion. Nascent replication tracts were IdU and CIdU-labelled before treatment with aphidicolin and replication fork stalling. Label retention (CIdU:IdU ratio) was assessed as a measure of fork stability. $V h l^{-/-}$MEFs displayed a significant reduction of median CIdU:IdU tract ratios (Fig. 2d and Supplementary Fig. 4e), indicating that VHL loss induces fork instability and collapse, an effect consistent with the observed depletion of RAD51 and RPA cellular pools.

PBRM1 loss induces H3K9me3-marked chromatin reorganisation. Though RAD51 and RPA remain significantly reduced in double-mutant cells, these cells display no replication fork defects (Fig. 2b, d). Furthermore, we did not observe any significant 
a

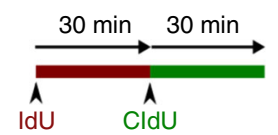

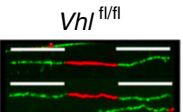

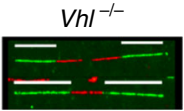

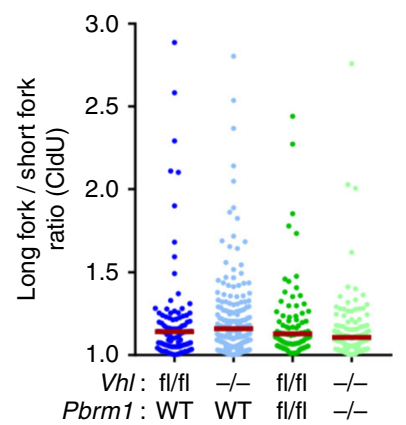

b
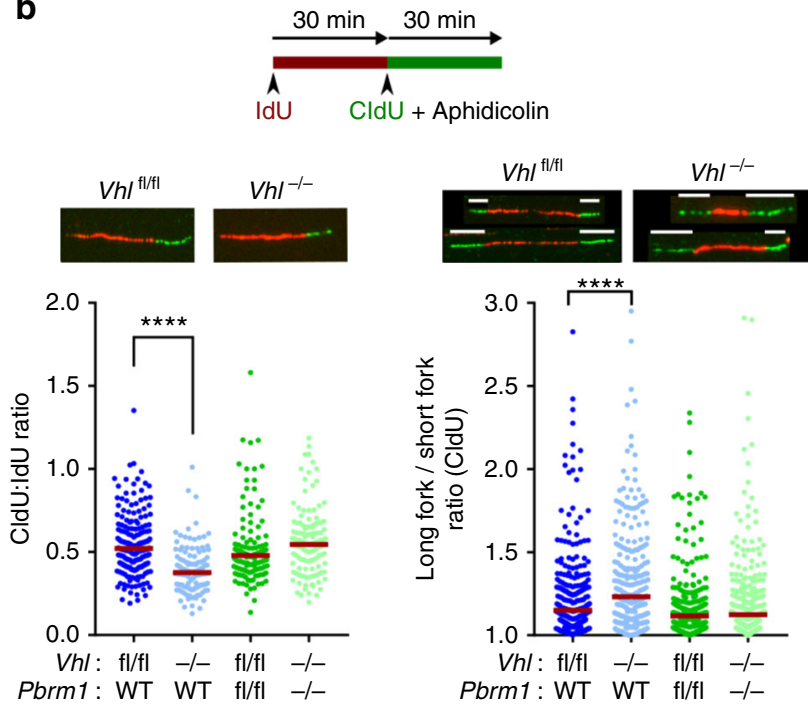

d
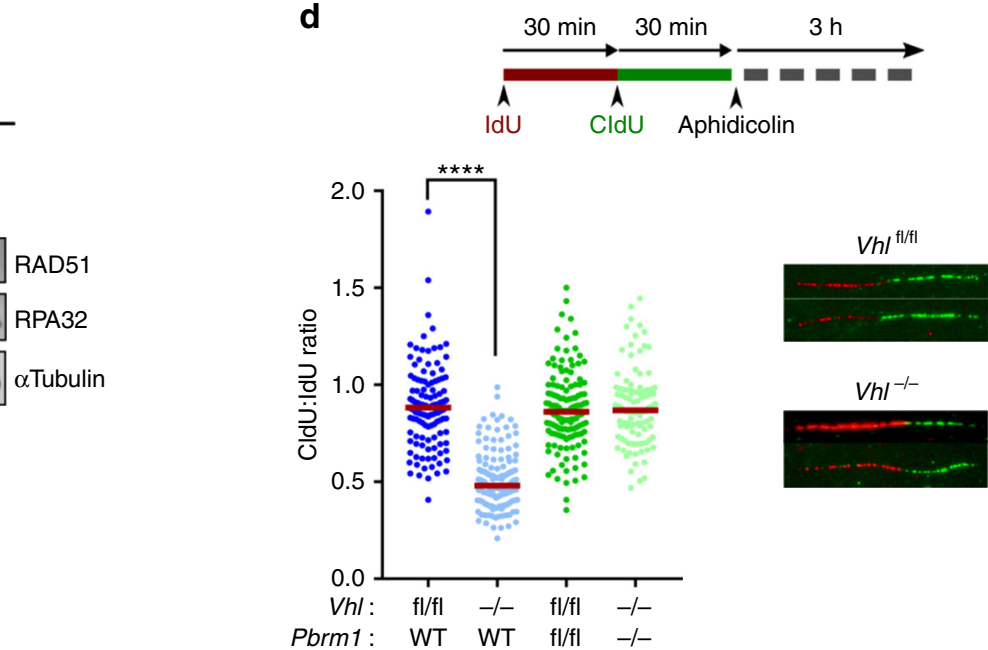

Fig. 2 Loss of VHL induces replication fork stalling and collapse. $\mathbf{a}, \mathbf{b}$ DNA fibre analyses following the indicated treatments. Top: DNA fibre assay experimental design. Bottom left: Representative images of labelled replication tracts and dot plot of CldU:IdU track ratio in MEFs of indicated genotype (data from representative experiment; $n>85$ tracts analysed for each genotype per experiment, $n$ (independent experiments) $=3$ ). Bottom right: Representative images of replication forks (white tracts indicate the lengths of the bidirectional forks analysed) and quantification of replication fork asymmetry ( $n>50$ forks in total per genotype, data pooled from $n$ (independent experiments) $=3$ ). c Representative immunoblots of MEFs. $\mathbf{d}$ Top: Experimental design of fork protection assay. Bottom left: Dot plot of of CIdU:IdU track ratio in MEFs (data from representative experiment, $n>220$ tracts analysed for each genotype per experiment, $n$ (independent experiments) $=3$ ). Bottom right: Representative images of labelled replication tracts. $\mathbf{a}, \mathbf{b}, \mathbf{d}$ Median values shown in red; Kruskall-Wallis, Dunnet's correction ${ }^{\star \star \star \star} p<0.0001$

differences in the activation of DNA damage responses in PBRM1 depleted cells (Supplementary Fig. 4f), suggesting that PBRM1 loss bypasses VHL-induced replication stress through an alternative pathway. PBRM1 encodes BAF180, a component of the SWI/SNF chromatin-remodeller complex PBAF, capable of modulating DNA/chromatin interactions ${ }^{37}$. Recent studies have shown that the chromatin context in which replication stress occurs can strongly influence DNA damage responses, with the preferential enrichment of $\gamma \mathrm{H} 2 \mathrm{AX}$ in heterochromatic regions marked by $\mathrm{H} 3 \mathrm{~K} 9 \mathrm{me}^{31}{ }^{31}, 38,39$. Furthermore, the modulation of chromatin structure and specifically $\mathrm{H} 3 \mathrm{~K} 9 \mathrm{me} 3$ is able to modify DNA replication, restore repair capacity and suppress DNA damage ${ }^{38-40}$. We, therefore, ascertained the chromatin localisation of DNA damage observed following MMC in $\mathrm{Vhl}^{-/-}$MEFs and found that $\gamma \mathrm{H} 2 \mathrm{AX}$ foci were preferentially associated with H3K9me3-marked chromatin (Fig. 3a). We thus hypothesised that PBRM1 loss could alleviate replication stress by modifying chromatin structure.

We first examined the distribution of nuclear $\mathrm{H} 3 \mathrm{~K} 9 \mathrm{me} 3$ in MEFs. Although we detected no change in the total levels of cellular $\mathrm{H} 3 \mathrm{~K} 9 \mathrm{me} 3$ between different genotypes, there was a striking difference in its distribution, with $\mathrm{Pbrm}^{-/-}$and $\mathrm{Vhl}^{-/-}$; $\mathrm{Pbrm1}^{-/-}$MEFs displaying more diffuse staining patterns and a significant reduction in the number of distinct foci (Fig. $3 \mathrm{~b}$ and Supplementary Fig. 5a). Furthermore, examination of H3K9me3 in the kidneys of control, $\mathrm{Vhl}^{-/-}, \mathrm{Pbrm}^{-/-}$and $\mathrm{Vhl}^{-/-} ; \mathrm{Pbrm} 1$ $-/-$ mice revealed a similar shift in distribution patterns, with an overall reduction in the number of discernible foci in mice lacking PBRM1 (Fig. 3c and Supplementary Fig. 5b). The effect of PBRM1 loss on chromatin structure appeared to be specific to $\mathrm{H} 3 \mathrm{~K} 9 \mathrm{me} 3$ with no differences observed in other heterochromatin markers (Supplementary Fig. 5c,d). 

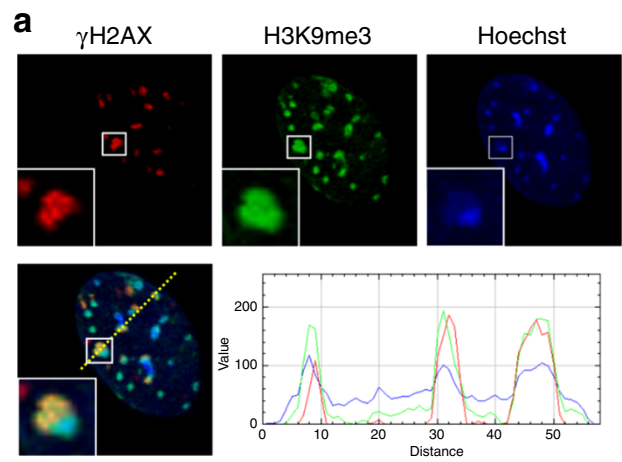

Merge

RGB profile

C
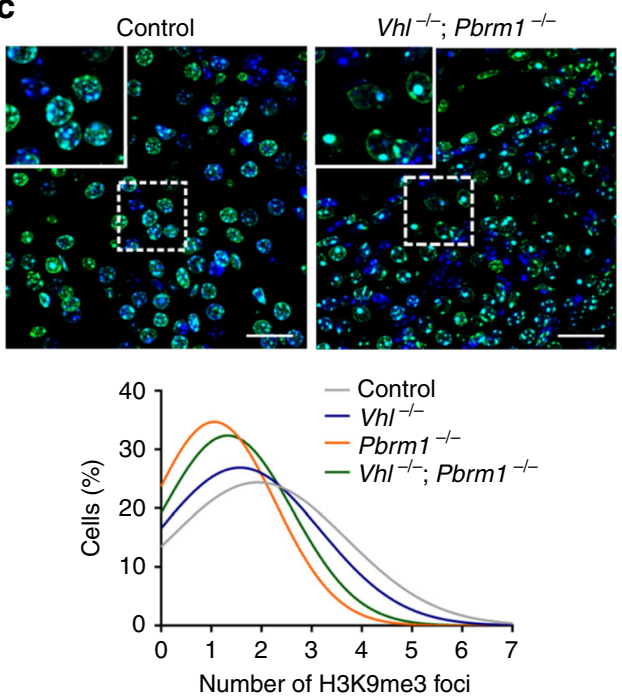

b
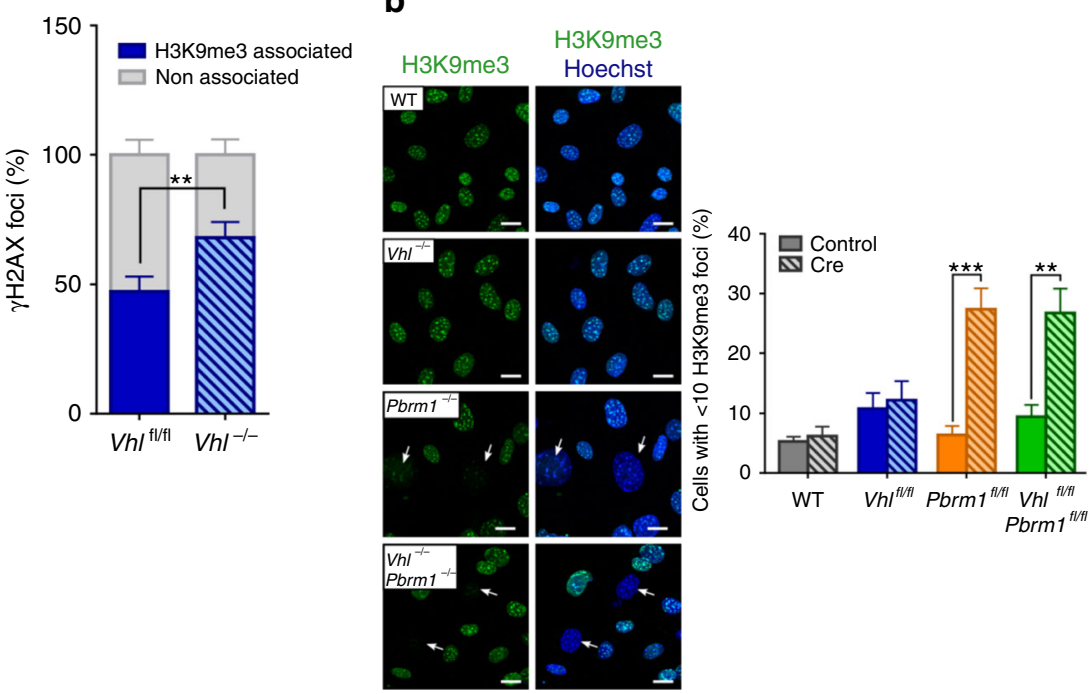

d
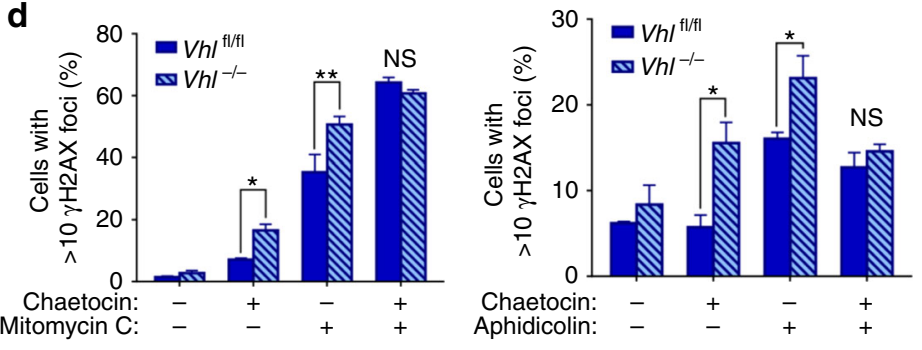

Fig. 3 PBRM1 loss reorganises H3K9me3-marked heterochromatin to reverse VHL-induced replication stress. a Left: representative images of immunofluorescence staining of $\gamma \mathrm{H} 2 \mathrm{AX}$ and $\mathrm{H} 3 \mathrm{~K} 9 \mathrm{me} 3$ in $\mathrm{Vhl}$-deleted MEFs $\left(\mathrm{Vh}^{-/-}\right)$at $24 \mathrm{~h}$ after Mitomycin $\mathrm{C}$. Line scans of $\gamma \mathrm{H} 2 \mathrm{AX}$, H3K9me3 and Hoechst signal intensity are presented in the panels below. Right: quantification of $\gamma \mathrm{H} 2 \mathrm{AX}$ and $\mathrm{H} 3 \mathrm{~K} 9 \mathrm{me} 3$ foci association ( $n$ (independent experiments) $=$ 3). b Left: representative images of $\mathrm{H} 3 \mathrm{~K} 9 \mathrm{me} 3$ immunofluorescence in day $8 \mathrm{MEFs}$. Arrows indicate cells with indistinct $\mathrm{H} 3 \mathrm{~K} 9 \mathrm{me} 3 \mathrm{foci}$. Scale bars, 20 $4 \mathrm{~m}$. Right: Quantification of H3K9me3 foci (n (independent experiments) =5). c H3K9me3 immunofluorescence in the kidneys of control and mutant mice. Representative images are shown in the top panels and histogram distributions of the number of $\mathrm{H} 3 \mathrm{~K} 9 \mathrm{me} 3$ foci per cell are shown in the bottom ( $n=4$ mice per genotype; at least 6000 cells analysed per animal). Scale bars, $100 \mu \mathrm{m}$. d Quantification of $\gamma \mathrm{H} 2 \mathrm{AX}$ foci formation at $24 \mathrm{~h}$ following treatment of MEFs as indicated ( $n$ (independent experiments) $=3$ ). Graphs depict mean \pm s.e.m. (error bars). (a, d) Two-tailed paired $t$-test. (b) Two-way ANOVA, Sidak's correction. ${ }^{\star \star \star} P<0.001 ;{ }^{\star \star} P<0.01 ;{ }^{\star} P<0.05$; NS not significant

To ascertain whether $\mathrm{H} 3 \mathrm{~K} 9 \mathrm{me} 3$ modulation is able to rescue VHL-dependent replication stress, $\mathrm{Vhl}^{-/-} \mathrm{MEFs}$ were treated with the histone lysine methyltransferase inhibitor chaetocin ${ }^{41}$ at doses sufficient to reduce the number of distinct $\mathrm{H} 3 \mathrm{~K} 9 \mathrm{me} 3$ nuclear foci without depleting modification levels (to recapitulate loss of PBRM1; Supplementary Fig. 5e-g). Treatment with chaetocin was able to reverse the previously observed, VHLinduced MMC and APH hypersensitivity (Fig. 3d) suggesting that the rescue of VHL-induced replication stress by PBRM1 is mediated at least in part by its effects on $\mathrm{H} 3 \mathrm{~K} 9 \mathrm{me} 3$ organisation.

PBRM1 loss restores the cellular fitness of VHL-deleted cells. Replication stress and the ensuing DNA damage induce checkpoint activation and cell cycle arrest, thereby curtailing cellular proliferation and cancer growth ${ }^{42}$. We therefore hypothesised that loss of PBRM1 and subsequent rescue of the DNA damage caused by $V H L$ deletion will confer a cellular fitness advantage to these cells. Though primary $\mathrm{Vhl}^{-/-}$and $\mathrm{Vhl}^{-/-} ; \mathrm{Pbrml}^{-/-}$MEFs showed no difference in their proliferation rates under unperturbed culture conditions, we observed a differential response following treatment with MMC, with the reduced viability of $V h l$ $-/$ cells but not the double mutants (Fig. $4 \mathrm{a}$ ).

The inducible nature of our genetically modified mouse models allows induction of genetic changes upon a defined time point (tamoxifen administration) thereby permitting observation and fate determination of recombined cells over time. To assess in vivo proliferative differences we quantified the allelic frequency of the recombined $V h l$ allele $\left(V h l^{-}\right)$in young ( $<12$ months) and aged (>20 months) $\mathrm{Vhl}^{-/-}$and $\mathrm{Vhl}^{-/-} ; \mathrm{Pbrm}^{-/-}$mutant mice. Renal $V h l^{-}$allelic content did not vary with age in $V h l^{-/-}$mice (Fig. 4b). In contrast, $\mathrm{Vhl}^{-/} ; \mathrm{Pbrm}^{-/-}$mice showed a 5-fold increase in $\mathrm{Vhl}$ - allelic content with increasing age, indicating that double-mutant epithelia gain a significant proliferative advantage over $\mathrm{Vhl}$-deleted cells. The expansion of double-mutant epithelial cells was further confirmed by CA9 immunohistochemistry (a marker of VHL loss) demonstrating a clear increase in the number of positively staining double-mutant epithelia over time (Fig. 4c). No such change was observed in Vhl-deleted animals.

These observations support our hypothesis that loss of PBRM1 improves the cellular fitness of VHL-defective cells permitting cellular growth and enhancing their proliferative capacity. 
a
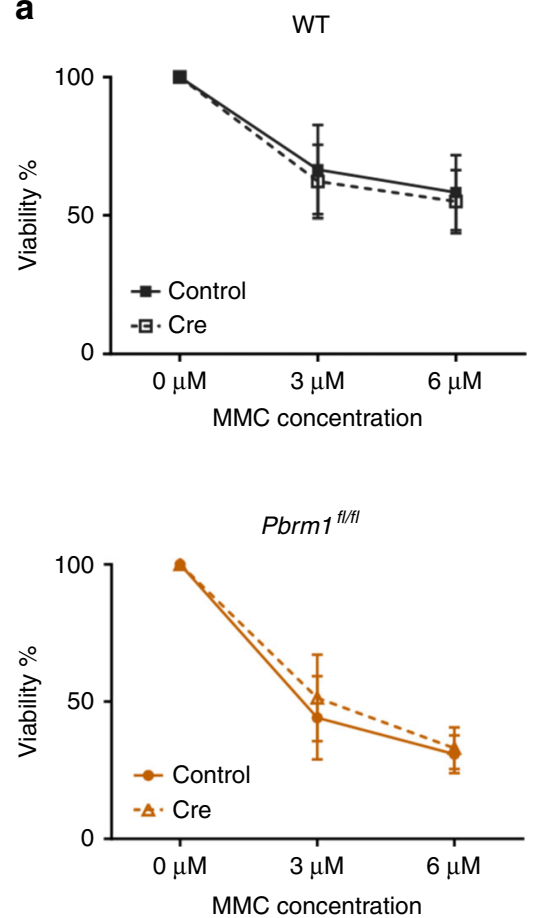

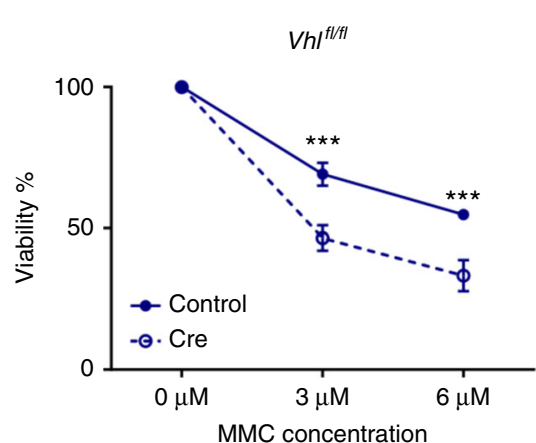

b
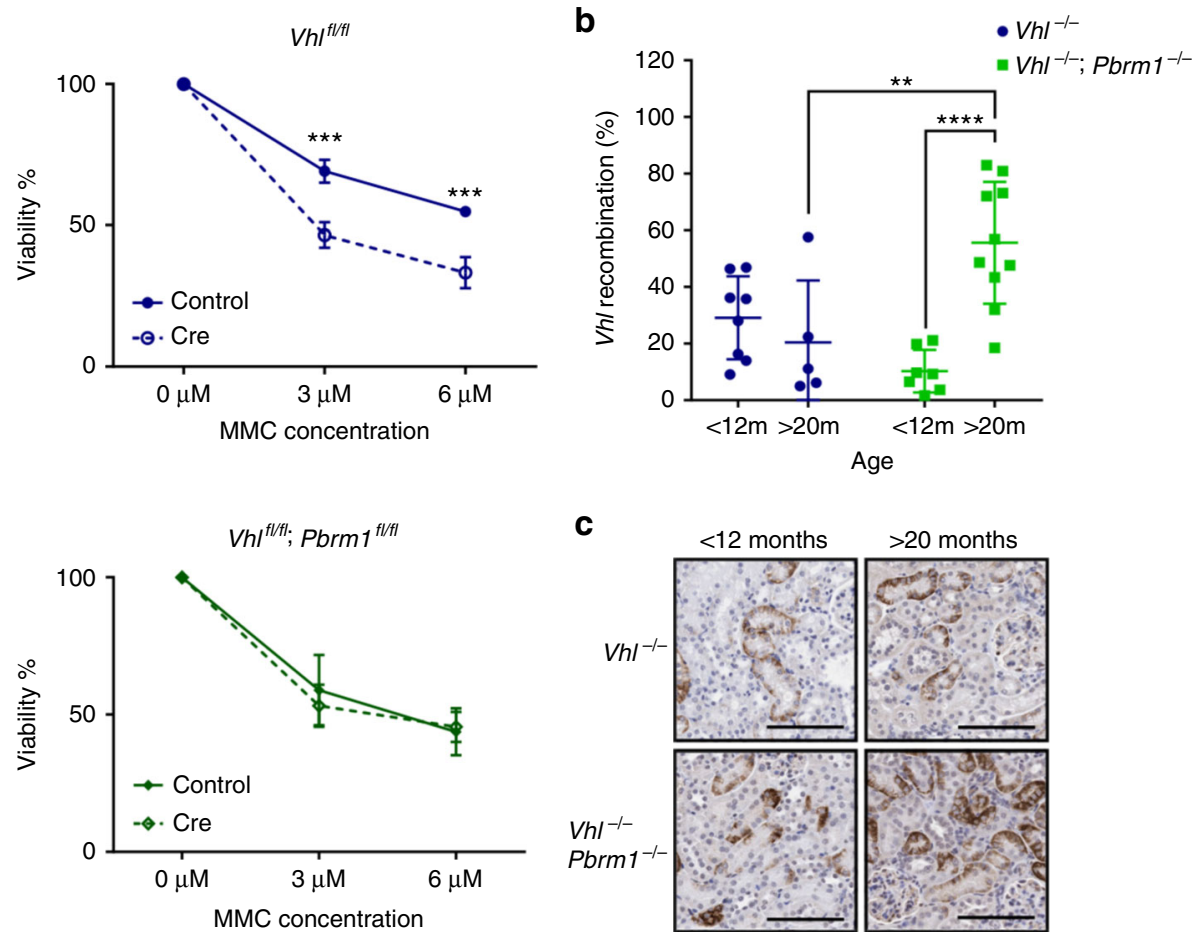

C

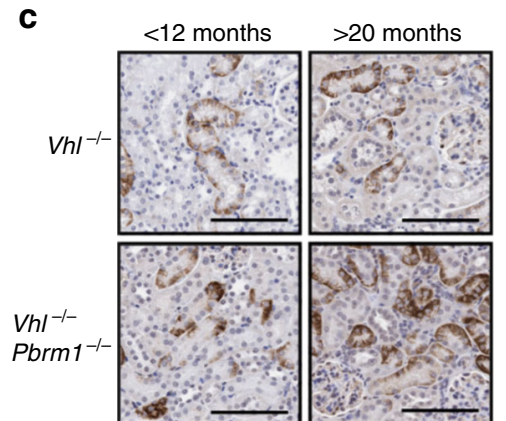

Fig. 4 Combined loss of VHL and PBRM1 improves cellular fitness. a MEF viability at 6 days following exposure to Mitomycin $\mathrm{C}$ ( $n$ (independent

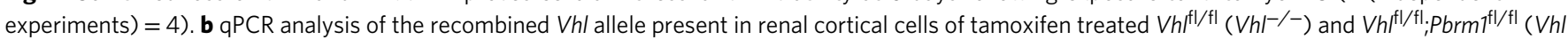

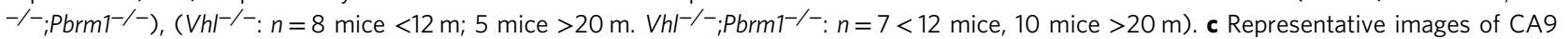
immunohistochemistry of $\mathrm{Vhl}^{-1-}$ and $\mathrm{Vhl}^{-1-} ; \mathrm{Pbrm}^{-/-}$mice at indicated ages. Graphs depict mean \pm s.e.m. (error bars). (a) Two-way ANOVA, Sidak's correction. (b) Two-way ANOVA, Tukey's correction. ${ }^{\star \star \star \star} P<0.0001 ;{ }^{\star \star \star} P<0.001 ;{ }^{\star \star} P<0.01$

$\mathrm{Vhl}^{-/-} ; \mathbf{P b r m 1}^{-/-}$mice develop clear cell RCCs. To ascertain whether biallelic deletion of $\mathrm{Vhl}$ and $\mathrm{Pbrm} 1$ is indeed sufficient to promote renal transformation, $\mathrm{Vhl}^{-/-}, \mathrm{Pbrml}^{-/-}$and $\mathrm{Vhl}^{-/-}$; $\mathrm{Pbrm}^{-/-}$mice as well as control animals were aged and observed for tumour development.

No renal tumours were observed in any of the aged control, $\mathrm{Vhl}^{-/-}$or Pbrm $1^{-/-}$animals. In contrast, $100 \%$ of $\mathrm{Vhl}^{-/-} ; \mathrm{Pbrm} 1$ $-/-$ mice developed renal cancers by 20 months of age (Fig. 5a and Supplementary Fig. 6a). Double-mutant mice displayed a spectrum of premalignant and malignant lesions ranging from simple, benign cysts (with one to two cell layers of bland epithelium), to atypical cysts (demonstrating epithelial hyperplasia with or without mild cytological atypia) and multifocal neoplastic tumours (Fig. 5b and Supplementary Fig. 6b). Importantly, lesions arose within microscopically normal renal cortical parenchyma as is commonly observed in the sporadic cases of human ccRCC. No macroscopic metastases were observed in any of the mice. All renal lesions displayed carbonic anhydrase 9 (CA9) positivity, a diagnostic marker of human clear cell RCC and VHL inactivation ${ }^{43}$. Vhl and Pbrml were confirmed to be homozygously deleted both by PCR and immunohistochemical analysis of neoplastic lesions (Fig. $5 c$ and Supplementary Fig. 6c), indicating that these arose from double-mutant epithelia. The $\mathrm{Vhl}^{-/-} ; \mathrm{Pbrm}^{-/-}$tumours displayed solid growth patterns with prominent vasculature and were composed of cells with eosinophilic or clear cytoplasm, and increased proliferative index, features reminiscent of the histopathological appearances of human ccRCC (Fig. 5d, e and Supplementary Fig. 6d). Renal tubular marker immunohistochemistry (megalin, Tamm-Horsfall protein (THP) and aquaporin 2, for proximal, distal tubules and collecting ducts, respectively) revealed all lesions staining strongly positive for megalin but no other renal markers, suggesting they originated from epithelia of proximal renal tubules
(Supplementary Fig. 6e). Altogether, these data indicate that the combined loss of $\mathrm{Vhl}$ and Pbrm1 within mouse tubular epithelia is sufficient for the development of renal neoplasias closely resembling the human disease.

\section{Discussion}

Here we report the generation of a novel genetically and clinically relevant mouse model of sporadic renal cell carcinoma and provide for the first time mechanistic data shedding light into the early events driving renal carcinogenesis and the essential tumour suppressor role of PBRM1. Our data shows that loss of VHL induces replication stress and DNA damage accumulation, responses that curtail cellular proliferation and transformation. Significantly, we find that loss of PBRM1, through modulation of H3K9me3-marked heterochromatin, rescues VHL-dependent replication stress and confers a survival and proliferative advantage, thereby permitting renal carcinogenesis (Fig. 5f).

Replication stress is a complex phenomenon that has serious implications for genome stability, cell survival and human disease. It has being increasingly recognised as a hallmark of precancerous and early neoplastic lesions that leads to genomic instability and activation of DDR responses leading to apoptosis or senescence, thereby curtailing their growth ${ }^{26}$. In ccRCC replication stress has been observed in the absence of SETD2 and is shown to enhance genomic instability and heterogeneity, impacting on cancer progression ${ }^{44}, 45$. Our data now establish replication stress as an early, underlying defect in the vast majority of ccRCCs, driven by the loss of $V H L$ and its effects on replication fork progression and stability. Both the observed depletion of cellular RAD51 and RPA levels, two proteins essential for the protection and restart of replication forks 33,34 , as well as the induction of replication stress have been previously 
associated with conditions of hypoxia ${ }^{30,31}$, suggesting that these are likely to represent HIF-dependent responses. Importantly, our results provide a biological explanation for the lack of transformation upon loss of VHL alone and the need for additional genetic hits to initiate renal carcinogenesis.
To overcome oncogene-induced replication stress and allow tumour progression, a second, essential step in cancer development involves suppression of the DDR responses, commonly achieved through mutations targeting TP53, ATM or MDM2 genes $^{26}$. Mutations of these genes though commonly observed in a
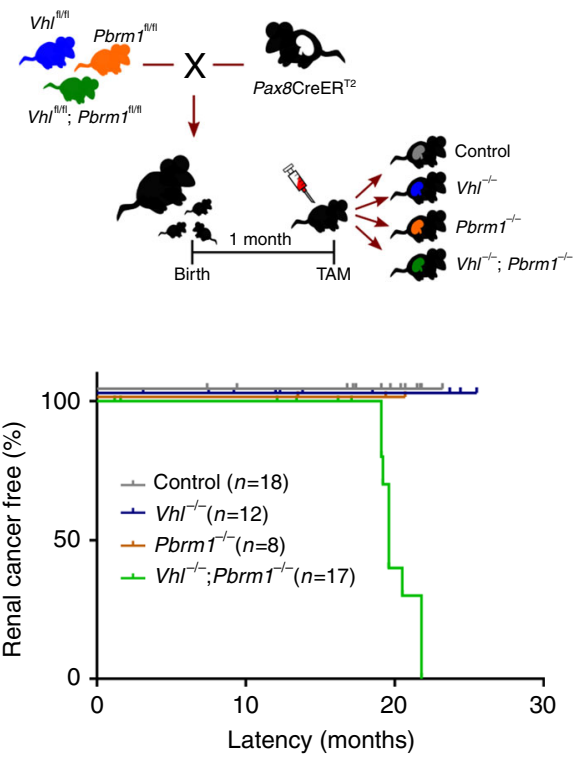

C

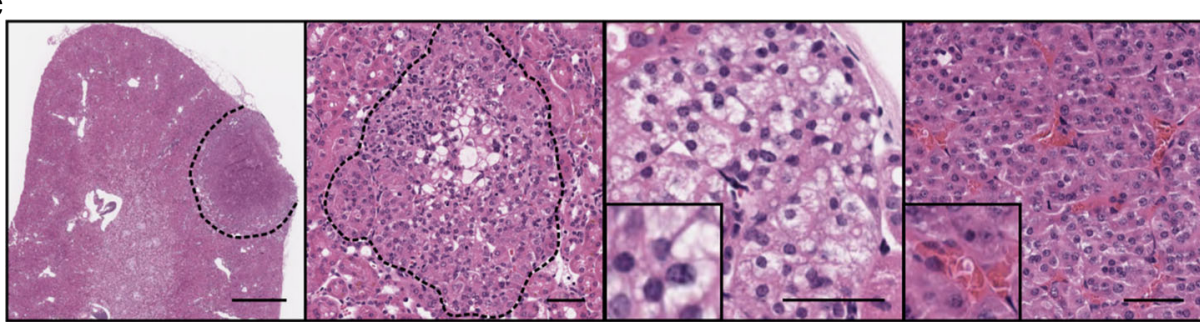

d

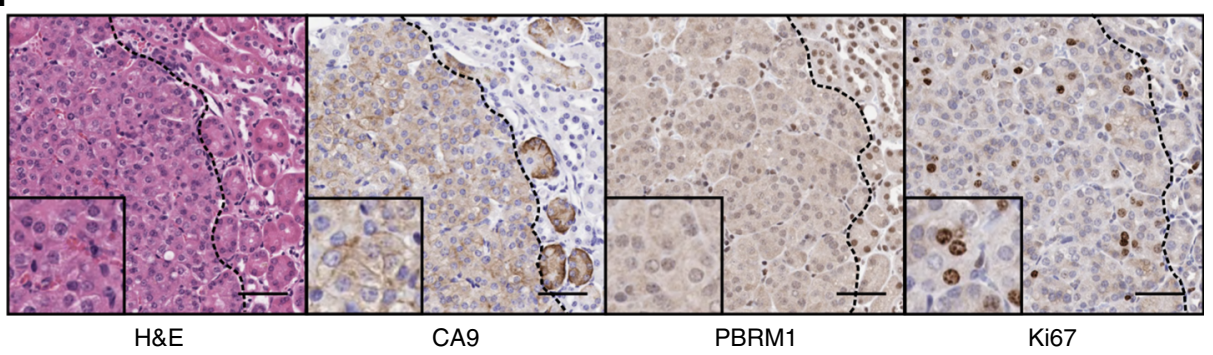

e
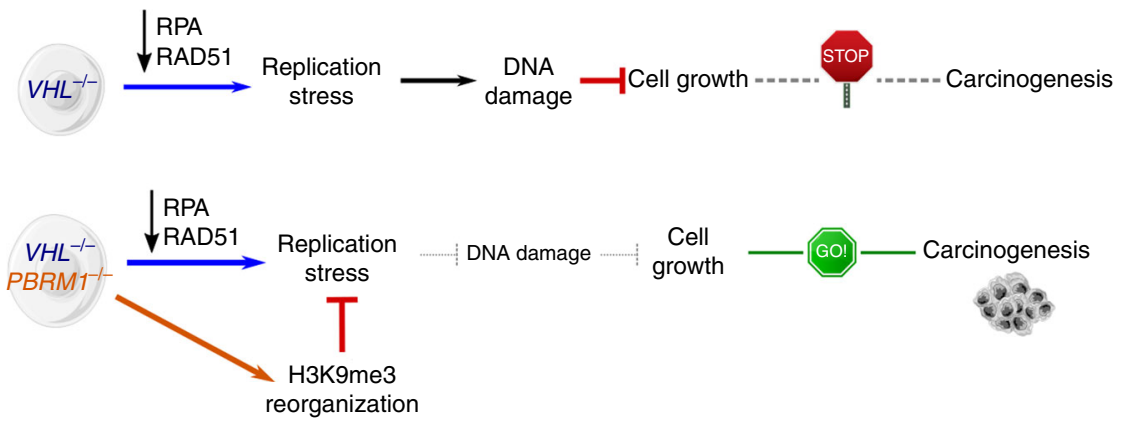
other tumour types are rare in $\mathrm{ccRCC}^{46}$. Our data shows that in the case of ccRCC, rescue of the replication stress response can be achieved through loss of the SWI/SNF chromatin remodeller PBRM1 and appears to be mediated, at least in part, through changes in $\mathrm{H} 3 \mathrm{~K} 9 \mathrm{me} 3$-marked heterochromatin. H3K9me3 has been previously shown to play a critical role in linking chromatin structure to replication and the DDR response ${ }^{31,40,47}$. Furthermore, modulation of $\mathrm{H} 3 \mathrm{~K} 9 \mathrm{me} 3$ can rescue the DNA repair defects observed with ATM deficiency ${ }^{38}$ as well as the damage accumulation and early senescence associated with progeria ${ }^{39}$. The mechanisms underlying these observations are currently unclear but are thought to involve modulation of DDR responses. Therefore, one mechanism by which loss of heterochromatin organisation (in the absence of PBRM1) could rescue the VHLinduced replication defects is through alterations in DDR signalling. Though we see no evidence of a significant defect in the activation of DNA damage responses upon PBRM1 loss, further detailed investigation in future studies is required to precisely define the molecular interactions underlying these findings. Whatever the precise mechanism of replication stress rescue, both our in vitro and in vivo data clearly show that loss of PBRM1 restores the cellular fitness and proliferative potential of $\mathrm{Vhl}$ deleted cells and is therefore critical for renal transformation.

SWI/SNF genes are commonly mutated across multiple cancer types and recent studies have reported their role in controlling chromatin structure and subsequent gene expression for the suppression of tumour development ${ }^{48-50}$. Our findings identify additional tumour suppressive functions mediated by SWI/SNF genes beyond the control of gene expression and highlight the interaction of chromatin structure and replication stress in initiating carcinogenesis. As a number of histone modifiers have been implicated in ccRCC development it will be of great interest to establish their precise interactions with VHL loss and the ensuing replication stress response.

Finally, we report the development of a novel genetically and clinically relevant mouse model of ccRCC representative of the sporadic form of the disease. While this manuscript was under preparation two groups reported the generation of mice with targeted disruption of $V h l$ and Pbrml in the kidney ${ }^{51,52}$. Nargund et al. ${ }^{51}$ induced $\mathrm{Vhl}$ and $\mathrm{Pbrm} 1$ deletion using a traditional Ksp-Cre driver with mice developing renal hydronephrosis, polycystic kidneys, renal failure and occasional renal tumours (observed in $30 \%$ of mice). In the second study, Gu et al. ${ }^{52}$ utilised the same promoter as used here (Pax8) to drive constitutive expression of Cre recombinase from early kidney development, with mice developing renal cancers of similar histological appearances to the ones observed in our model. In contrast to these studies, our use of Pax8-CreER ${ }^{T 2}$ transgene allows for temporal and spatial control of $V h l$ and Pbrml deletion and, therefore, the specific targeting of fully developed renal tubular epithelium. Thereby our study provides proof that ccRCC can arise from the fully differentiated epithelium of renal proximal tubules. Furthermore, the inducible nature of our model avoids potential confounding by effects of $\mathrm{Vhl}$ and Pbrm 1 deletion during renal embryogenesis (as observed in the other RCC mouse models). As a consequence, the tumours observed develop within normal surrounding renal parenchyma and without signs of morbidity, representing an accurate model of early, sporadic human ccRCC and therefore a useful tool for the discovery and preclinical evaluation of new methodologies for early detection, prevention and treatment of the disease. Importantly, our study provides essential insights into the molecular mechanisms underpinning renal carcinogenesis, highlighting replication stress as an underlying vulnerability for the majority of ccRCC and providing opportunities for the development of much needed novel therapeutics.

\section{Methods}

Data reporting. No statistical methods were used to predetermine sample size. The experiments were not randomised and the investigators were not blinded to allocation during experiments. For outcome assessment most experiments were automatically analysed by Fiji scripts to minimise potential human bias. When manual annotation was required, blinding precautions were made.

Mouse strains and treatments. $P a x 8 C r e E R^{T 2}$ transgenic mice have been previously described ${ }^{16}$. Conditional $V h f^{f l f l}$ mice were obtained from Jackson Laboratories (B6.129S4(C)-VhlmlJae $/ J)^{53}$. Pbrm fllfl $^{\text {mice }}$ were generated by the Wellcome Trust Sanger Institute (Hinxton, UK), using European Conditional Mouse Mutagenesis Program (EUCOMM) targeted embryonic stem cells (Pbrm1 1mla(EUCOMM)Wtsi. clone ID EPD0089_3_C01). Briefly, a knockout-first conditional allele composed of an IRES:lacZ trapping cassette and a floxed promoter-driven neomycin cassette flanking exon 4 of the Pbrm1 gene (Extended Data Fig. 3a), was targeted in C57BL/6 N embryonic stem cells ${ }^{54}$. The EPD0089_3_C01 clone was passaged twice in $2 \mathrm{i} / \mathrm{LIF}$ media ${ }^{55}$ at low density to enrich for ground state pluripotent cells. ES cell clones were isolated and expanded for injection into 8-cell embryos to produce chimeric founders. Genotyped progeny that were positive for germline transmission were bred, and first generation offspring that inherited the targeted allele were subsequently mated with FLPeR mice $^{56}$ to remove the lacZ/neomycin selection cassette, leaving loxP sites on either side of exon 4 (exon 2 of the translated transcript). The FLPeR allele was bred out of the mouse line following cassette elimination. All mice were maintained on a C57BL/6 background. Mice were crossbred to produce experimental cohorts

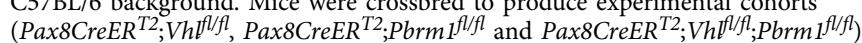
and littermate controls ( $V h \ell^{f l / f l}, P b r m 1^{f l / f l}$ and $\left.V h l^{f l / f l} ; P b r m 1^{f l / f l}\right)$. Mouse genotypes from ear biopsies were determined using real-time PCR with specific probes designed for each gene (Transnetyx, Cordova, TN). To induce deletion of target genes 4-week to 6-week-old experimental and control animals were injected intraperitoneally with $2 \mathrm{mg}$ of tamoxifen (Sigma, UK) for 5 consecutive days ${ }^{16}$.

For the mitomycin C (MMC) sensitivity studies, cohorts of mice 2-6 months post tamoxifen induction (3-8 months of age) were treated with either saline or MMC $(0.5 \mathrm{mg} / \mathrm{ml}$; Sigma, UK) at a single intraperitoneal injection of $5 \mathrm{mg} / \mathrm{kg}$. Mice were euthanized 10 days post treatment and kidneys were collected for further analyses. Seven out of $39(18 \%)$ animals showed macroscopic and microscopic signs of renal cortical glomerular and tubular atrophy, a previously reported toxicity of MMC in mice ${ }^{57}$. Affected mice were not of particular sex, age or genotype (4/20 controls, 2/8 Pax8CreER ${ }^{T 2} ; V_{h} f^{f l / f l}$ and $1 / 4$ Pax $8 \mathrm{CreER}^{T 2} ; \mathrm{Pbrm}^{\mathrm{fl} / \mathrm{fl}}$ ) and were excluded from further analysis.

Animal studies were conducted using age-matched littermate controls for each experiment. Both male and female mice were used. Animals were maintained under specific pathogen free conditions in accordance to Home Office UK regulations and the Animals (Scientific Procedures) Act, 1986. All experimental protocols were approved by the Animal Welfare and Ethical Review Body (AWERB) of the University of Cambridge CRUK Cambridge Institute.

Generation and culture of MEFs. MEFs were isolated from WT, $\mathrm{Vhl}^{\mathrm{f} / / \mathrm{fl}}, \mathrm{Pbrm}^{\mathrm{fl} / \mathrm{fl}}$ and $\mathrm{Vhl}^{\mathrm{fl} / \mathrm{fl}} ; \mathrm{Pbrm} 1^{\mathrm{fl} / \mathrm{fl}}$ animals at embryonic day 12.5 and aliquots were frozen at passage 3. Cells were cultured in DMEM supplemented with 10\% FBS (Thermo Fisher) and $1 \%$ penicillin/streptomycin (Thermo Fisher) and maintained in conventional cell culture incubators at $5 \%$ oxygen to replicate physiological tissue conditions ${ }^{20}$. For Cre-mediated recombination of target genes, two days after thawing, MEFs were treated for two consecutive days with $1 \mu \mathrm{M}$ of TAT-Cre Recombinase (SCR508, Merck Millipore) and $100 \mu \mathrm{M}$ of Chloroquine (C6628, Sigma) for $5 \mathrm{~h}$; control cells were treated with $100 \mu \mathrm{M}$ of Chloroquine only.

Fig. 5 Combined loss of $V h l$ and Pbrm1 is sufficient to initiate renal carcinogenesis. a Top: schematic of crossings, tamoxifen (TAM) administration and resulting cohorts. Bottom: Kaplan-Meier renal cancer-free survival of control and mutant mice. b Representative Haematoxylin and Eosin (H\&E) and CA9

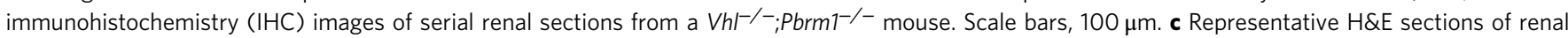
neoplasias from $\mathrm{Vhl}^{-/-} ; \mathrm{Pbrm}^{-/-}$mice demonstrating cytoplasmic clearing (middle panels) and prominent thin-walled vasculature (right panel). Scale bar in left panel, $1 \mathrm{~mm}$. Other scale bars, $50 \mu \mathrm{m}$. d Images of serial sections of a representative renal tumour from a $\mathrm{Vh}{ }^{-/-} ; \mathrm{Pbrm}^{-/-}$mouse stained with $\mathrm{H} \& \mathrm{E}^{-}$ or following immunohistochemical analyses with the indicated antibodies. Scale bars, $50 \mu \mathrm{m}$. e Schematic interpretation of the effects of VHL loss alone or in combination with PBRM1 and their contributions to renal transformation 
Treatment was repeated at day 15 after recombination to ensure cultures remained fully recombined. Recombination was confirmed by PCR and immunoblotting. Data were obtained from at least three independent experiments using primary MEFs derived from a minimum of three different embryos and two separate primary cultures. For proliferation assays, cells were seeded in 12-well plates at 40,000 cells $/ \mathrm{ml}$ in triplicate wells and counted after 4 days, before re-seeding at the same density for the next passage. Cumulative population doubling was calculated as log $\left(\mathrm{N}_{\mathrm{f}} / \mathrm{N}_{\mathrm{i}}\right) / \log _{2}$, where $\mathrm{N}_{\mathrm{i}}$ and $\mathrm{N}_{\mathrm{f}}$ correspond to number of cells plated and final counts/passage, respectively.

All cells tested negative for mycoplasma contamination.

Immunoblotting. MEFs were lysed in RIPA buffer with protease and phosphatase inhibitors and lysates were processed using standard methods. We used the following primary antibodies: VHL (sc-5575, 1:250; Santa Cruz), PBRM1 (A301590A, 1:1000; Bethyl Laboratories), alpha-Tubulin (T5168, 1:2000; Sigma), RAD51 (sc-8349, 1:100; Santa Cruz), RPA32 (A300-244A, 1:500; Bethyl Laboratories), pATM (ab36810, 1:1000; Abcam), ATM (ab85213, 1:1000; Abcam), pDNA-PKc (ab18192, 1:1000; Abcam), DNA-PKc (ab70250, 1:1000; Abcam), pCHK1 (\#2348 S, 1:500; Cell Signalling), CHK1 (\#2360, 1:1000; Cell Signalling), H3K9me3 (ab8898, 1:1000; Abcam), H3K27me3 (ab6002, 1:1000; Abcam), H3K9me2 (ab8898, 1:1000; Abcam), H4K20me3 (ab9053, 1:1000; Abcam), HPla (ab77256, 1:1000, Abcam), KAP1 (ab10484, 1:1000; Abcam) and H3 (4499, 1:2000; Cell Signalling). Secondary antibodies were conjugated to IRDye 680 or 800 (Li-Cor). Fluorescent signals were imaged using the Odyssey Infrared Imaging System (Li-Cor). Western blot band quantifications were performed with Fiji ${ }^{58}$. Uncropped scans of presented blots are shown in Supplementary Fig. 7.

Hypersensitivity assays. MEFs were seeded at 40,000 cells/ml on Ibidi 8 -well $\mu$ Slides (IB-80826, Ibidi) and allowed to attach for $48 \mathrm{~h}$ before treatment. All cells were treated with either mitomycin C (MMC) or aphidicolin (APH) at day 6 post recombination. For MMC assays cells were treated with $2 \mu \mathrm{M}$ of MMC (Sigma) for $2 \mathrm{~h}$ before replacing with either fresh media or media containing $200 \mathrm{nM}$ of CDKi (CDK1/2 Inhibitor III, 217714, Merck Chemicals) as specified. Alternatively, cells were treated with $75 \mathrm{nM}$ of Chaetocin for $24 \mathrm{~h}$ before adding $2 \mu \mathrm{M}$ of MMC for $2 \mathrm{~h}$. After treatment, fresh media containing $75 \mathrm{nM}$ of Chaetocin was added for a further $24 \mathrm{~h}$. For APH assays, aphidicolin (A4487, Sigma) was added at $200 \mathrm{nM}$ with or without $200 \mathrm{nM}$ of CDKi for $24 \mathrm{~h}$. Alternatively, cells were treated with 75 $\mathrm{nM}$ of Chaetocin for $24 \mathrm{~h}$ before adding aphidicolin $200 \mathrm{nM}$ to the media for a further $24 \mathrm{~h}$

Immunocytochemistry. MEFs grown on Ibidi 8-well $\mu$ Slides were washed with PBS and fixed in $4 \%$ paraformaldehyde for $15 \mathrm{~min}$. For pATR and pCHK1 detection, cells were permeabilized by treating them with CSK buffer $(20 \mathrm{mM}$ Tris, $300 \mathrm{mM}$ glucose, $100 \mathrm{mM} \mathrm{NaCl}, 3 \mathrm{mM} \mathrm{MgCl} 2,0.5 \%$ Triton X-100) for $12 \mathrm{~min}$ at room temperature followed by incubation with CSK-S buffer $(10 \mathrm{mM}$ Tris, $10 \mathrm{mM}$ $\mathrm{NaCl}, 3 \mathrm{mM} \mathrm{MgCl} 2,1 \%$ Tween, $0.5 \% \mathrm{SDS}$ ) for $12 \mathrm{~min}$. For other antibodies, cells were permeabilized with $0.5 \%$ Triton X-100 in PBS for 10 min. Cells were then blocked in $2.5 \%$ normal donkey serum (NDS) for $1 \mathrm{~h}$ and incubated with primary antibody diluted in $1 \%$ NDS overnight at $4{ }^{\circ} \mathrm{C}$ in a humid box. Cells were incubated with Alexa fluor fluorochrome (Thermo Fisher) secondary antibodies diluted in 1\% NDS for $30 \mathrm{~min}$ at room temperature before being incubated with Hoechst 33258 $(1 \mu \mathrm{g} / \mathrm{mL}$, Thermo Fisher) for $5 \mathrm{~min}$. The wells were washed with PBS and covered with a drop of Mounting media (Ibidi). We used the following primary antibodies: $\gamma \mathrm{H} 2 \mathrm{AX}$ (9718, 1:1,000; Cell Signalling and ab26350, 1:500; Abcam), 53BP1 (IHC00001, 1:500; Bethyl Laboratories), pATR (\#2853, 1:500; Cell Signalling), pCHK1 (\#2348S, 1:50, Cell Signalling), H3K9me3 (ab8898, 1:1000; Abcam), H2AZ (ab4174, 1:100; Abcam), H3K27me3 (ab6002, 1:500; Abcam), H3K9me2 (ab8898, 1:200; Abcam), H4K20me3 (ab9053, 1:100; Abcam), HP1a (ab77256, 1:200; Abcam) and KAP1 (ab10484, 1:500; Abcam). Image stacks of whole cells were obtained by confocal laser-scanning microscopy (Leica TCS SP5) and automatic counting of foci and nuclei detection was performed by an appropriate macro in Fiji. Association of $\gamma \mathrm{H} 2 \mathrm{AX}$ with $\mathrm{H} 3 \mathrm{~K} 9 \mathrm{me} 3$ foci was analysed using Imaris software (Bitplane). In all studies more than 100 cells were analysed per genotype for each experiment.

$\boldsymbol{\beta}$-galactosidase staining. Cells were seeded at a density of $80,000 \mathrm{cells} / \mathrm{ml}$ in $12-$ well plate 2 days prior the staining for $\beta$-galactosidase. At day 20 after recombination cells were washed with PBS and $\beta$-galactosidase activity was assessed using the $\beta$-galactosidase staining kit (9860, Cell signalling) following the manufacture's protocol. Images were taken with NIKON Eclipse TS100 microscope and processed with Fiji software for analysis. More than 150 cells were evaluated per genotype for each experiment.

DNA fibre assays. Asynchronous MEFs were labelled as indicated with $50 \mu \mathrm{M}$ iododeoxyuridine (IdU, Sigma I7125) followed by $500 \mu \mathrm{M}$ of chlorodeoxyuridine (CldU, Sigma C6891). Aphidicolin (200 nM) was added with the second pulse of CldU or immediately after labelling with IdU as indicated. Cells were collected and resuspended in PBS at a density of $1 \times 10^{6}$ cells $/ \mathrm{mL}$. $2 \mu \mathrm{L}$ of cell suspension was spotted onto a Superfrost microscope slide (Thermo Fisher) and overlaid with $7 \mu \mathrm{L}$ of DNA lysis buffer (200 mM Tris-HCl, $50 \mathrm{mM}$ EDTA, 0.5\% SDS). After $10 \mathrm{~min}$, the slides were tilted by 15 degrees to allow lysates to slowly move down the slide. The DNA spreads were air-dried for $4 \mathrm{~h}$, fixed in a 3:1 mixture of methanol/acetic acid for $10 \mathrm{~min}$ at room temperature and air-dried overnight. The slides were then treated with $2.5 \mathrm{~N} \mathrm{HCl}$ for $60 \mathrm{~min}$ at $37^{\circ} \mathrm{C}$, washed in PBS and incubated in blocking buffer (5\% BSA/PBS) for $30 \mathrm{~min}$ at room temperature followed by incubation with a mouse anti-BrdU antibody (to detect IdU) (347590, 1:50, BD Biosciences) and a rat anti-BrdU antibody (to detect CldU) (ab6326, 1:200, Abcam). After washing three times with PBS, the slides were incubated for $12 \mathrm{~min}$ in stringency buffer ( $36 \mathrm{mM}$ Tris- $\mathrm{HCl}, 0.5 \mathrm{mM} \mathrm{NaCl}$ and $0.5 \%$ Tween), rinsed three times in PBS and incubated for 30 min with Alexa Fluor 488-conjugated antirat and Alexa Fluor 555-conjugate anti-mouse, washed in PBS and mounted in Mounting media (Ibidi). Fibre images were obtained in a Leica DMI 6000B microscope using a $63 \mathrm{x}$ objective. In each assay $>100$ fibres were measured and each individual experiment was repeated at least two times.

Real-time PCR. Total RNA was isolated from MEFs using the RNeasy Mini kit (Qiagen). Purified RNA was reverse transcribed using the SuperScript II Reverse Transcriptase kit (Thermo Fisher). Rad51 and RPA32 levels were measured using the TaqMan gene expression assays and a Quantstudio 6 Instrument (Applied Biosystems). Real-time quantitative PCRs were carried out following the manufacture's protocol using the following probes: Mm00488047_m1 (RPA2), Mm00487905_m1 (Rad51), Mm00437762_m1 (B2M), Mm03024075_m1 (HPRT) Relative sample quantification was carried out by the comparative DDCt method using B2M and HPRT as endogenous controls.

Viability assays. At day 4 after recombination MEFs were seeded at 20,000 cells/ $\mathrm{ml}$ in triplicate in 96-well plates and allowed to attach for $48 \mathrm{~h}$ before treatment. Mitomycin $\mathrm{C}$ was added to cells for $2 \mathrm{~h}$ at the stated concentrations before medium was replaced with fresh growth medium. Viability was analysed 6 days following treatment using MTT assay (CGD1, Sigma) according to the manufacturer's protocol.

Histology and immunohistochemistry. At experimental end-points animals were euthanized and tissues were fixed in $4 \%$ paraformaldehyde in PBS overnight and embedded in paraffin. To review histology, $4 \mu \mathrm{m}$ sections were stained with haematoxylin and eosin (H\&E). Immunohistochemistry (IHC) and immunofluorescence (IF) were performed on $4 \mu \mathrm{m}$ tissue sections using standard protocols. Specificity of immunostaining was assessed by incubation in the absence of primary or secondary antibody. We used the following primary antibodies: $\gamma \mathrm{H} 2 \mathrm{AX}(9718$, 1:1000; Cell Signalling), H3K9me3 (ab8898, 1:500; Abcam), p21 (sc-471, 1:2000; Santa Cruz), ki67 (NCL-ki67p, 1:1000; Novacastra), PBRM1 (A301-591A, 1:2000; Bethyl Laboratories), CA9 (sc-25600, 1:200; Santa Cruz), aquaporin 2 (ab105171, 1:1000; Abcam), megalin (ab76969, 1:1000; Abcam) and Tamm-Horsfall protein (THP; AF5175, 1:1000; R\&D Systems). Secondary antibodies used were conjugated to HRP (IHC) or Alexa fluor fluorochromes (IF). Fluorescent images were obtained by confocal laser-scanning microscopy (Leica TCS SP5). All histology was independently reviewed by a specialist renal pathologist.

For quantification of $\gamma \mathrm{H} 2 \mathrm{AX}, \mathrm{p} 21$ and ki67 expression, stained tissue sections were scanned with the Aperio ScanScope (Aperio, Vista, CA) and images were visualised and captured using the Aperio ImageScope program. Quantification was performed on a minimum of 15 fields ( $\times 20$ magnification) from three representative coronal kidney sections per animal. Images were processed using Fiji software by calculating the percentage of DAB positive nuclei relative to total number of nuclei within the field (a minimum of 8000 cells were analysed per animal).

For quantification of $\mathrm{H} 3 \mathrm{~K} 9 \mathrm{me} 3$, a minimum of 10 image stacks were obtained from at least 2 coronal sections of kidney per animal by confocal laser-scanning microscopy (Leica TCS SP5). Nuclei detection and foci counting was performed automatically by an appropriate macro in FIJI. At least 6000 cells were analysed per animal. For quantification of $\gamma \mathrm{H} 2 \mathrm{AX} / \mathrm{CA} 9$ co-immunostaining, a minimum of six image stacks were obtained from at least two coronal sections of kidney per animal by confocal laser-scanning microscopy (Leica TCS SP5). Staining was quantified automatically by an appropriate macro in FIJI. At least 10,000 cells were analysed per animal.

Quantitative PCR. Genomic DNA from macroscopically normal renal cortical parenchyma was extracted using standard protocols. For quantitative PCR (qPCR) of the Vhl deletion, SYBR Green PCR Master Mix (Applied Biosystems) was used and amplification and analysis was carried out on QuantStudio 6 (Thermo Fisher Scientific). Primers forward 5'-CTGGTACCCACGAAAGTGTC-3' and reverse $5^{\prime}$ CTGACTTCCACTGATGCTTGTCACAG-3' were used to detect the recombined allele $\left(V h l^{-}\right)$. Serial dilutions of fully recombined $V h l^{-1-}$ MEF DNA were used to construct a standard curve and to determine $\mathrm{Vhl}^{-}$allelic frequency.

Genotyping. Genotyping of MEFs and additional genotyping of mice was performed by PCR using the following primers and conditions. $V h l$ allele recombination: forward primer for floxed allele $5^{\prime}$-CCGGAGTAGGATAAGTCAGCTGAG-3', forward primer for recombined allele 5'-CTGGTACCCACGAAAGTGTC-3', common 
reverse primer 5'-CTGACTTCCACTGATGCTTGTCACAG-3' (400 bp product for floxed allele, $200 \mathrm{bp}$ product for $\mathrm{WT}$ and $250 \mathrm{bp}$ product for recombined allele); 1 cycle of $10 \min 94^{\circ} \mathrm{C}, 55$ cycles of $\left(50 \sec 95^{\circ} \mathrm{C}, 50 \sec 58^{\circ} \mathrm{C}, 60 \sec 72^{\circ} \mathrm{C}\right), 1$ cycle of $5 \mathrm{~min} 72^{\circ} \mathrm{C}^{16}$. Cre recombinase: forward primer $5^{\prime}$-GCACTGATTTCGACCAGGTT-3', reverse primer 5'-GCTAACCAGCGTTTTCGTTC-3' (200 bp product); Pbrm 1 allele recombination: forward primer $5^{\prime}$ GCAGGAGAGTTATATAAGCCAATAAGCTG-3', reverse primer 5'-TGACACCAAAGAGTTCTCCAGGATCA-3' (1266 bp product for floxed allele, $1119 \mathrm{bp}$ product for WT and $423 \mathrm{bp}$ product for recombined allele); 1 cycle of $1 \mathrm{~min} 95^{\circ} \mathrm{C}, 30$ cycles of $\left(15 \mathrm{~s} 95^{\circ} \mathrm{C}, 30 \mathrm{~s} 58^{\circ} \mathrm{C}, 1 \mathrm{~min} 68^{\circ} \mathrm{C}\right), 1$ cycle of $5 \mathrm{~min} 68^{\circ} \mathrm{C}$.

Statistical analysis. Data were visualised and statistical analyses performed using Prism 7.0 software (Graph Pad). $P<0.05$ was considered statistically significant. For cases where experimental groups showed comparable variance the following statistical tests were applied. $P$-values for unpaired comparisons between two groups were calculated by two-tailed Student's $t$-test, whilst $P$-values for paired comparisons were calculated by two-tailed paired $t$-test. One-way analysis of variance (ANOVA) was used for analysis between three or more groups, followed by appropriate multiple comparison correction. Ordinary two-way ANOVA was used for analysis that involved two variables, followed by appropriate multiple comparison correction. For experiments showing non-comparable variance between groups the Kruskall-Wallis test was applied followed by Dunn's correction for multiple comparisons. ${ }^{*} P<0.05 ;{ }^{* *} P<0.01 ;{ }^{* *} P<0.001 ;{ }^{* *} P<0.0001$. Error bars, mean \pm s.d. or s.e.m., as indicated.

Data availability. The data that support the findings of this study are available within the Article and Supplementary Files, or available from the authors upon request.

Received: 27 March 2017 Accepted: 15 November 2017 Published online: 11 December 2017

\section{References}

1. Ferlay, J. et al. GLOBOCAN 2012 v1.0, Cancer Incidence and Mortality Worldwide: IARC CancerBase No. 11, http://globocan.iarc.fr (2013).

2. Motzer, R. J., Hutson, T. E., McCann, L., Deen, K. \& Choueiri, T. K. Overall survival in renal-cell carcinoma with pazopanib versus sunitinib. N. Engl. J. Med. 370, 1769-1770 (2014).

3. Gossage, L., Eisen, T. \& Maher, E. R. VHL, the story of a tumour suppressor gene. Nat. Rev. Cancer 15, 55-64 (2015).

4. Latif, F. et al. Identification of the von Hippel-Lindau disease tumor suppressor gene. Science 260, 1317-1320 (1993).

5. Gnarra, J. R. et al. Mutations of the VHL tumour suppressor gene in renal carcinoma. Nat. Genet 7, 85-90 (1994).

6. Herman, J. G. et al. Silencing of the VHL tumor-suppressor gene by DNA methylation in renal carcinoma. Proc. Natl Acad. Sci. USA 91, 9700-9704 (1994).

7. Nickerson, M. L. et al. Improved identification of von Hippel-Lindau gene alterations in clear cell renal tumors. Clin. Cancer Res. 14, 4726-4734 (2008).

8. Zbar, B., Brauch, H., Talmadge, C. \& Linehan, M. Loss of alleles of loci on the short arm of chromosome 3 in renal cell carcinoma. Nature 327, 721-724 (1987).

9. Beroukhim, R. et al. Patterns of gene expression and copy-number alterations in von-hippel lindau disease-associated and sporadic clear cell carcinoma of the kidney. Cancer Res. 69, 4674-4681 (2009).

10. Cancer Genome Atlas Research. Comprehensive molecular characterization of clear cell renal cell carcinoma. Nature 499, 43-49 (2013).

11. Gerlinger, M. et al. Genomic architecture and evolution of clear cell renal cell carcinomas defined by multiregion sequencing. Nat. Genet. 46, 225-233 (2014).

12. Metcalf, J. L. et al. K63-ubiquitylation of VHL by SOCS1 mediates DNA double-strand break repair. Oncogene 33, 1055-1065 (2014).

13. Mandriota, S. J. et al. HIF activation identifies early lesions in VHL kidneys: evidence for site-specific tumor suppressor function in the nephron. Cancer Cell 1, 459-468 (2002).

14. Varela, I. et al. Exome sequencing identifies frequent mutation of the SWI/SNF complex gene PBRM1 in renal carcinoma. Nature 469, 539-542 (2011).

15. Rankin, E. B., Tomaszewski, J. E. \& Haase, V. H. Renal cyst development in mice with conditional inactivation of the von Hippel-Lindau tumor suppressor. Cancer Res. 66, 2576-2583 (2006).

16. Espana-Agusti, J. et al. Generation and characterisation of a Pax8-CreERT2 transgenic line and a Slc22a6-CreERT2 knock-in line for inducible and specific genetic manipulation of renal tubular epithelial cells. PLoS One 11, e0148055 (2016).
17. Fu, L., Wang, G., Shevchuk, M. M., Nanus, D. M. \& Gudas, L. J. Generation of a mouse model of Von Hippel-Lindau kidney disease leading to renal cancers by expression of a constitutively active mutant of HIFlalpha. Cancer Res. 71, 6848-6856 (2011).

18. Schietke, R. E. et al. Renal tubular HIF-2alpha expression requires VHL inactivation and causes fibrosis and cysts. PLoS One 7, e31034 (2012).

19. Fu, L., Wang, G., Shevchuk, M. M., Nanus, D. M. \& Gudas, L. J. Activation of HIF2alpha in kidney proximal tubule cells causes abnormal glycogen deposition but not tumorigenesis. Cancer Res. 73, 2916-2925 (2013).

20. Aukland, K. \& Krog, J. Renal oxygen tension. Nature 188, 671 (1960).

21. Parrinello, S. et al. Oxygen sensitivity severely limits the replicative lifespan of murine fibroblasts. Nat. Cell Biol. 5, 741-747 (2003).

22. Welford, S. M., Dorie, M. J., Li, X., Haase, V. H. \& Giaccia, A. J. Renal oxygenation suppresses VHL loss-induced senescence that is caused by increased sensitivity to oxidative stress. Mol. Cell. Biol. 30, 4595-4603 (2010).

23. Brownlee, P. M., Chambers, A. L., Cloney, R., Bianchi, A. \& Downs, J. A. BAF180 promotes cohesion and prevents genome instability and aneuploidy. Cell Rep. 6, 973-981 (2014).

24. Hell, M. P., Duda, M., Weber, T. C., Moch, H. \& Krek, W. Tumor suppressor VHL functions in the control of mitotic fidelity. Cancer Res. 74, 2422-2431 (2013).

25. Kakarougkas, A. et al. Requirement for PBAF in transcriptional repression and repair at DNA breaks in actively transcribed regions of chromatin. Mol. Cell $\mathbf{5 5}$ 723-732 (2014)

26. Macheret, M. \& Halazonetis, T. D. DNA replication stress as a hallmark of cancer. Annu. Rev. Pathol. 10, 425-448 (2015).

27. Tannenbaum, M. Ultrastructural pathology of human renal cell tumors. Pathol. Аnпu. 6, 249-277 (1971)

28. Kragel, P. J., Walther, M. M., Pestaner, J. P. \& Filling-Katz, M. R. Simple renal cysts, atypical renal cysts, and renal cell carcinoma in von Hippel-Lindau disease: a lectin and immunohistochemical study in six patients. Mod. Pathol. 4, 210-214 (1991).

29. Motzer, R. J., Bander, N. H. \& Nanus, D. M. Renal-cell carcinoma. N. Engl. J. Med. 335, 865-875 (1996).

30. Scanlon, S. E. \& Glazer, P. M. Multifaceted control of DNA repair pathways by the hypoxic tumor microenvironment. DNA Repair 32, 180-189 (2015).

31. Olcina, M. M. et al. Replication stress and chromatin context link ATM activation to a role in DNA replication. Mol. Cell 52, 758-766 (2013).

32. Leszczynska, K. B. et al. Mechanisms and consequences of ATMIN repression in hypoxic conditions: roles for p53 and HIF-1. Sci. Rep. 6, 21698 (2016).

33. Hashimoto, Y., Ray Chaudhuri, A., Lopes, M. \& Costanzo, V. Rad51 protects nascent DNA from Mre11-dependent degradation and promotes continuous DNA synthesis. Nat. Struct. Mol. Biol. 17, 1305-1311 (2010).

34. Toledo, L. I. et al. ATR prohibits replication catastrophe by preventing global exhaustion of RPA. Cell 155, 1088-1103 (2013).

35. Somyajit, K., Saxena, S., Babu, S., Mishra, A. \& Nagaraju, G. Mammalian RAD51 paralogs protect nascent DNA at stalled forks and mediate replication restart. Nucleic Acids Res. 43, 9835-9855 (2015).

36. Wang, G. et al. PTEN regulates RPA1 and protects DNA replication forks. Cell Res. 25, 1189-1204 (2015).

37. Thompson, M. Polybromo-1: the chromatin targeting subunit of the PBAF complex. Biochimie 91, 309-319 (2009).

38. Goodarzi, A. A. et al. ATM signaling facilitates repair of DNA double-strand breaks associated with heterochromatin. Mol. Cell 31, 167-177 (2008).

39. Liu, B. et al. Depleting the methyltransferase Suv39h1 improves DNA repair and extends lifespan in a progeria mouse model. Nat. Commun. 4, 1868 (2013)

40. Wu, R., Wang, Z., Zhang, H., Gan, H. \& Zhang, Z. H3K9me3 demethylase $\mathrm{Kdm} 4 \mathrm{~d}$ facilitates the formation of pre-initiative complex and regulates DNA replication. Nucleic Acids Res. 45, 169-180 (2017).

41. Greiner, D., Bonaldi, T., Eskeland, R., Roemer, E. \& Imhof, A. Identification of a specific inhibitor of the histone methyltransferase SU(VAR)3-9. Nat. Chem. Biol. 1, 143-145 (2005).

42. Halazonetis, T. D., Gorgoulis, V. G. \& Bartek, J. An oncogene-induced DNA damage model for cancer development. Science 319, 1352-1355 (2008).

43. Tostain, J., Li, G., Gentil-Perret, A. \& Gigante, M. Carbonic anhydrase 9 in clear cell renal cell carcinoma: a marker for diagnosis, prognosis and treatment. Eur. J. Cancer 46, 3141-3148 (2010).

44. Kanu, N. et al. SETD2 loss-of-function promotes renal cancer branched evolution through replication stress and impaired DNA repair. Oncogene 34, 5699-5708 (2015).

45. Pfister, S. X. et al. Inhibiting WEE1 Selectively Kills Histone H3K36me3Deficient Cancers by dNTP Starvation. Cancer Cell 28, 557-568 (2015).

46. Turajlic, S., Larkin, J. \& Swanton, C. SnapShot: Renal Cell Carcinoma. Cell 163, 1556 (2015).

47. Sun, Y. et al. Histone $\mathrm{H} 3$ methylation links DNA damage detection to activation of the tumour suppressor Tip60. Nat. Cell Biol. 11, 1376-1382 (2009). 
48. Kadoch, C. et al. Dynamics of BAF-Polycomb complex opposition on heterochromatin in normal and oncogenic states. Nat. Genet. 49, 213-222 (2017).

49. Wang, X. et al. SMARCB1-mediated SWI/SNF complex function is essential for enhancer regulation. Nat. Genet. 49, 289-295 (2017).

50. Mathur, R. et al. ARID1A loss impairs enhancer-mediated gene regulation and drives colon cancer in mice. Nat. Genet. 49, 296-302 (2017).

51. Nargund, A. M. et al. The SWI/SNF protein PBRM1 restrains VHL-LossDriven clear cell renal cell carcinoma. Cell Rep. 18, 2893-2906 (2017).

52. Gu, Y. F. et al. Modeling renal cell carcinoma in Mice: Bap1 and Pbrm1 inactivation drive tumor grade. Cancer Discov. 7, 900-917 (2017).

53. Haase, V. H., Glickman, J. N., Socolovsky, M. \& Jaenisch, R. Vascular tumors in livers with targeted inactivation of the von Hippel-Lindau tumor suppressor. Proc. Natl Acad. Sci. USA 98, 1583-1588 (2001).

54. Skarnes, W. C. et al. A conditional knockout resource for the genome-wide study of mouse gene function. Nature 474, 337-342 (2011).

55 . Guo, G. et al. Klf4 reverts developmentally programmed restriction of ground state pluripotency. Development 136, 1063-1069 (2009).

56. Farley, F. W., Soriano, P., Steffen, L. S. \& Dymecki, S. M. Widespread recombinase expression using FLPeR (flipper) mice. Genesis 28, 106-110 (2000).

57. Molyneux, G. et al. The haemotoxicity of mitomycin in a repeat dose study in the female CD-1 mouse. Int. J. Exp. Pathol. 86, 415-430 (2005).

58. Schindelin, J. et al. Fiji: an open-source platform for biological-image analysis. Nat. Methods 9, 676-682 (2012).

\section{Acknowledgements}

This research was supported by Cancer Research UK (C37839/A12177; J.E.A, A.M.). A. W. was funded by the Cambridge Cancer Centre. S.K.C. and D.J.A. were supported by the Wellcome Trust Sanger Institute. We acknowledge the following core facilities at the University of Cambridge CRUK Cambridge Institute: The Biological Resource Unit, Histopathology, Microscopy, Genomics. We thank D. J. Winton, R. S. Houlston and R. J. Gilbertson for advice and manuscript discussions.

\section{Author contributions}

J.E.A. and A.M. designed, performed and analysed the MEF and mouse experiments and wrote the manuscript. A.W. performed the histology review. S.K.C. contributed to the generation of the PBRM1 floxed mice and provided reagents. D.J.A. provided reagents, critical insight and edited the manuscript.

\section{Additional information}

Supplementary Information accompanies this paper at https://doi.org/10.1038/s41467017-02245-1.

Competing interests: J.E.A and A.M are currently paid employees of AstraZeneca. The remaining authors declare no competing financial interests.

Reprints and permission information is available online at http://npg.nature.com/ reprintsandpermissions/

Publisher's note: Springer Nature remains neutral with regard to jurisdictional claims in published maps and institutional affiliations.

(c) Open Access This article is licensed under a Creative Commons Attribution 4.0 International License, which permits use, sharing, adaptation, distribution and reproduction in any medium or format, as long as you give appropriate credit to the original author(s) and the source, provide a link to the Creative Commons license, and indicate if changes were made. The images or other third party material in this article are included in the article's Creative Commons license, unless indicated otherwise in a credit line to the material. If material is not included in the article's Creative Commons license and your intended use is not permitted by statutory regulation or exceeds the permitted use, you will need to obtain permission directly from the copyright holder. To view a copy of this license, visit http://creativecommons.org/ licenses/by/4.0/.

(C) The Author(s) 2017 